\title{
Pulmonary Spread of Juvenile Laryngeal Papillomatosis: A Case Report
}

\author{
Chen-Long Li, ${ }^{1}$ and Tian-Yu Zhang ${ }^{1,2,}{ }^{*}$ \\ ${ }^{1}$ Department of Otolaryngology-Head and Neck Surgery, Eye and ENT Hospital, Fudan University, Shanghai, China \\ ${ }^{2}$ Hearing Medicine Key Laboratory, National Ministry of Public Health, Shanghai, China \\ "Corresponding author: Tian-Yu Zhang, Department of Otolaryngology-Head and Neck Surgery, Eye and ENT Hospital, Fudan University, Shanghai, China. Tel: \\ +86-2164377134383, Fax: +86-02164377151, E-mail: ty.zhang2006@aliyun.com \\ Received 2015 December 25; Revised 2016 April 24; Accepted 2016 May 08.
}

\begin{abstract}
Introduction: Recurrent respiratory papillomatosis (RRP) is usually a relatively benign disease but can have an aggressive clinical course. The primary treatment for laryngeal papillomatosis is endoscopic resection. However, there is no effective treatment for pulmonary spread of laryngeal papillomatosis.

Case Presentation: We describe a rare case of a 19-year-old man with pulmonary spread of laryngeal papillomatosis. The treatment regimen was surgical resection under general anesthesia using suspension laryngoscopy and endoscopy. The patient had been diagnosed with laryngeal papillomatosis and had undergone a tracheotomy when he was 18 months old. He underwent 17 endoscopic resections for laryngeal papillomatosis at intervals of 3 months to 1 year. At follow-up, the patient continued to have tracheal intubation and was not extubated. CT scans revealed multiple nodular and cavitary lesions in both lungs. Laryngoscopy performed when the patient was 18 years showed diffuse papillomata in the glottis and left ventricular fold.

Conclusions: This article presents a rare case of juvenile laryngeal papillomatosis associated with the human papilloma virus (HPV) HPV-11 and pulmonary spread. Intravenous cidofovir has oncogenic potential, but it has been used successfully to treat five patients with pulmonary spread of RRP. Intravenous cidofovir should be considered as salvage therapy.
\end{abstract}

Keywords: Recurrent Respiratory Papillomatosis, Laryngeal Tumors, Pulmonary Spread, Cidofovir

\section{Introduction}

Recurrent respiratory papillomatosis (RRP), which most often affects the larynx, is usually a relatively benign disease, but it can have an aggressive clinical course (1). It spreads through the respiratory tract. Laryngeal papillomatosis has a high rate of recurrence after endoscopic resection and is difficult to eradicate (2). The human papilloma virus (HPV) is found in the majority of patients with RRP, especially types HPV-6 and -11. HPV-11 causes more aggressive disease than HPV-6.

The primary treatment for laryngeal papillomatosis is endoscopic resection. However, there is no effective treatment for pulmonary spread of laryngeal papillomatosis (2, 3). This article describes a rare case of a 19-year-old man with pulmonary spread of laryngeal papillomatosis and presents a brief review of cidofovir for the treatment of the pulmonary spread of RRP.

\section{Case Presentation}

We describe a rare case of a 19-year-old man with pulmonary spread of laryngeal papillomatosis. The treatment regimen was surgical resection under general anesthesia using suspension laryngoscopy and endoscopy. The patient had been diagnosed with laryngeal papillomatosis after he was born in 1995 and had undergone a tracheotomy when he was 18 months old. In 2009, he underwent 13 endoscopic resections for laryngeal papillomatosis at 3month intervals. Routine preoperative chest radiographs showed no obvious abnormalities until June 2009, when chest radiographs revealed several nodular lesions in the right lower lung and a suspicious nodular lesion in the left lower lung.

In September 2010, the patient was transferred to our clinic for recurrent laryngeal papillomatosis. Chest radiographs showed multiple nodular and cavitary lesions in both lung fields, especially in the right side. There were diffuse papillomata in the glottis and right arytenoid and a small papilloma in the ventricular fold. Numerous subglottic papillomata were found near the tracheal stoma, which was blocking the trachea. Surgical resection with a microdebrider was performed under general anesthesia using suspension laryngoscopy and endoscopy. The type of HPV was HPV-11. The patient underwent surgery for RRP in November 2010, April 2011, and November 2012. During the last surgery, diffuse papillomata were observed in the glottis, subglottis, endotracheal, and bronchus. Endoscopic resection was performed to ensure airway patency. However, the patient refused to undergo a pulmonary biopsy.

At follow-up, the patient continued to have tracheal intubation and was not extubated. CT scans revealed multiple nodular and cavitary lesions in both lungs (Figure 
1). Laryngoscopy performed when the patient was 18 years showed diffuse papillomata in the glottis and left ventricular fold (Figure 2). These findings suggested a diagnosis of pulmonary spread of laryngeal papillomatosis.
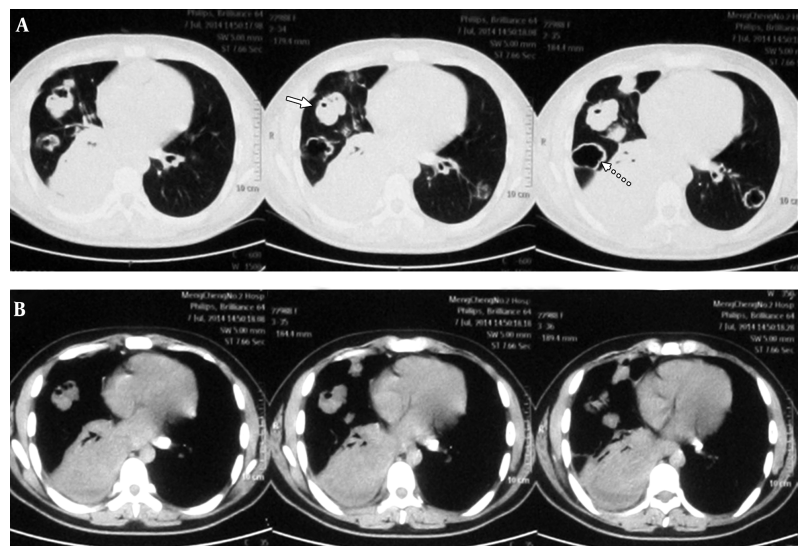

Figure 1. CT Scans Revealed Multiple Nodular and Cavitary Lesions (Arrows) in Both Lungs

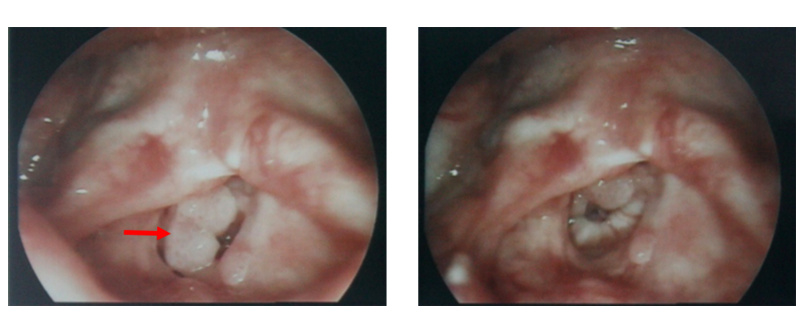

Figure 2. Laryngoscopy Showed Diffuse Papillomata (Arrows) in the Glottis and Left Ventricular Fold

\section{Discussion}

In RRP, the histology of papillomata is usually benign, and the papillomata are localized in the larynx. Lung involvement in RRP is rare, with a reported incidence of 3.3\% in cohort studies and a reported incidence of $16 \%$ in patients with cancer and lung involvement (2). Juvenile RRP primarily occurs in patients younger than 5 years, with $25 \%$ of these cases presenting during infancy. The juvenile form of RRP is considered more aggressive than the adult type (4). The present case was juvenile laryngeal papillomatosis.

HPV, especially types 6 and 11, are found in the majority of patients with RRP. In a a systematic review of lung involvement in juvenile onset RRP, researchers at the Montreal children's hospital performed HPV typing in 49 pa- tients, 27 ( $55 \%$ ) of whom subsequently developed lung cancer (2). Of these 27 patients, 18 had HPV-11, and only one patient had HPV-6. Patients with HPV-11 are prone to the development of more aggressive disease (2). In China, Yang reported one case of pulmonary spread of RRP associated with HPV-6, and the patient in that case developed lung cancer and died (5). The patient in the present case had HPV-11 and no lung cancer. The reason may be the difference between their susceptibility.

Interferon and cidofovir are specifically used to treat the pulmonary spread of RRP. According to a study by the Montreal children's hospital, interferon did not significantly change the course of RRP when the lungs were involved (2). However, Ablanedo-Terrazas et al. found that intralesional cidofovir therapy appeared to be effective in the treatment of RRP (6). Studies of 12 patients with pulmonary spread of RRP treated with intravenous cidofovir reported successful outcomes in five cases. Other drugs, including indole-3-carbinol and interferon- $\alpha$ (IFN- $\alpha$ ), have also been used to treat RRP. Dancey et al. reported the first successful case of a 35-year-old woman, with bilateral multiple pulmonary nodules and cysts, who was treated with subcutaneous IFN- $\alpha$-2a and intravenous cidofovir (7). Armbruster et al. reported a successful experience using a combination of intravenous cidofovir and IFN- $\alpha$-2b in a 34-year-old woman with RRP and bilateral round areas of cavitation and consolidation (8). Van Valckenborgh et al. reported the case of an 8-year-old girl who showed significant regression of lung lesions and no side effects after intravenous cidofovir therapy (9). De Bilderling reported one completely cured case of an 8-year-old girl, with suspected lung lesions of respiratory papillomatosis, who remained in remission for at least 2 years (10). The 8-yearold girl was treated with indole-3-carbinol, in conjunction with local and intravenous cidofovir, for 27 months. Riviere et al. reported the case of a 60-year-old woman with pulmonary spread of RRP who was treated with intravenous cidofovir only for 6 months (11). Intravenous cidofovir combatted RRP-related symptoms, as shown by CT scans and pulmonary function tests.

Cidofovir is a nucleoside analogue of deoxycytidine monophosphate. The latter cannot be used to treat RRP because of its potential oncogenicity. One study reported that cidofovir treatment failed in seven cases with RRP, and three cases developed squamous cell carcinomas (2). However, another study reported that cidofovir inhibited the polymerization of viral DNA and enhanced the hostmediated immune response (12). Given the possible oncogenic potential of intravenous cidofovir, it should only be considered as salvage therapy. At present, cidofovir is not available in China.

This paper described a rare case of pulmonary spread 
of juvenile laryngeal papillomatosis associated with HPV11. Although intravenous cidofovir has oncogenic potential, it was associated with successful outcomes in five patients with pulmonary spread of RRP. Intravenous cidofovir should only be considered as salvage therapy.

\section{Acknowledgments}

Dr Chen-long Li drafted the initial manuscript and reviewed and revised the manuscript; Dr Tian-yu Zhang conceptualized and designed the study and reviewed and revised the manuscript. Both authors approved the final submitted manuscript.

\section{References}

1. Boltezar IH, Bahar MS, Zargi M, Gale N, Maticic M, Poljak M. Adjuvant therapy for laryngeal papillomatosis. Acta Dermatovenerol Alp Pannonica Adriat. 2011;20(3):175-80. [PubMed: 22131118].

2. Gelinas JF, Manoukian J, Cote A. Lung involvement in juvenile onset recurrent respiratory papillomatosis: a systematic review of the literature. Int J Pediatr Otorhinolaryngol. 2008;72(4):433-52. doi: 10.1016/j.ijporl.2007.12.003. [PubMed: 18281102].

3. Soldatski IL, Onufrieva EK, Steklov AM, Schepin NV. Tracheal, bronchial, and pulmonary papillomatosis in children. Laryngoscope. 2005;115(10):1848-54. doi: 10.1097/01.mlg.0000173155.57491.2a. [PubMed: 16222208].

4. Katsenos S, Becker HD. Recurrent respiratory papillomatosis: a rare chronic disease, difficult to treat, with potential to lung cancer transformation: apropos of two cases and a brief literature review. Case Rep Oncol. 2011;4(1):162-71. doi: 10.1159/000327094. [PubMed: 21526134].
5. Xiao Y, Wang J, Han D, Ma L. A Case of the Intrapulmonary Spread of Recurrent Respiratory Papillomatosis With Malignant Transformation. Am J Med Sci. 2015;350(1):55-7. doi:10.1097/MAJ.0000000000000370. [PubMed: 25423295].

6. Ablanedo-Terrazas Y, Soda-Merhy A, Hernandez-Palestina M, Ormsby $\mathrm{CE}$, Reyes-Teran G. Intralesional cidofovir in severe juvenile respiratory papillomatosis. B-ENT. 2012;8(3):197-202. [PubMed: 23113383].

7. Dancey DR, Chamberlain DW, Krajden M, Palefsky J, Alberti PW, Downey GP. Successful treatment of juvenile laryngeal papillomatosis-related multicystic lung disease with cidofovir: case report and review of the literature. Chest. 2000;118(4):1210-4. [PubMed: 11035700].

8. Armbruster C, Kreuzer A, Vorbach H, Huber M, Armbruster C. Successful treatment of severe respiratory papillomatosis with intravenous cidofovir and interferon alpha-2b. Eur RespirJ. 2001;17(4):8301. [PubMed: 11401080].

9. Van Valckenborgh I, Wellens W, De Boeck K, Snoeck R, De Clercq E, Feenstra L. Systemic cidofovir in papillomatosis. Clin Infect Dis. 2001;32(3):E62-4. doi: 10.1086/318497. [PubMed: 11170974].

10. de Bilderling G, Bodart E, Lawson G, Tuerlinckx D, Remacle M, Naesens L, et al. Successful use of intralesional and intravenous cidofovir in association with indole-3-carbinol in an 8-year-old girl with pulmonary papillomatosis. J Med Virol. 2005;75(2):332-5. doi: 10.1002/jmv.20275. [PubMed: 15602729].

11. Riviere F, Gille T, Le Tinier JY, Gharbi N, Khalil A, Wislez M, et al. Treatment of recurrent respiratory papillomatosis lung involvement by cidofovir infusion. Scand J Infect Dis. 2011;43(2):112-4. doi: 10.3109/00365548.2010.530687. [PubMed: 21039305].

12. Shehab N, Sweet BV, Hogikyan ND. Cidofovir for the treatment of recurrent respiratory papillomatosis: a review of the literature. Pharmacotherapy. 2005;25(7):977-89. [PubMed: 16006276]. 